\title{
Prognostic significance of low TSH concentration in patients with COVID-19 presenting with non-thyroidal illness syndrome
}

Jing Gong ${ }^{1}$, Ding-kun Wang ${ }^{1}$, Hui Dong ${ }^{2}$, Qing-song Xia ${ }^{2}$, Zhao-yi Huang ${ }^{1}$, Yan Zhao ${ }^{1}$, Xing Chen ${ }^{3,4}$, Fen Yuan', Jing-bin $\mathrm{Li}^{\mathrm{i}^{* \dagger}}$ and Fu-er Lü ${ }^{2^{*+}}$

\begin{abstract}
Background: Low free triiodothyronine (FT3) levels are related to a poor prognosis deterioration in patients with COVID-19 presenting with non-thyroidal illness syndrome (NTI). This study was designed to explore whether free thyroxin (FT4) or thyroid stimulating hormone (TSH) levels affected the mortality of patients with COVID-19 presenting with NTI.

Methods: Patients with COVID-19 complicated with NTI who were treated at our hospital were included in this retrospective study. Patients were divided into low TSH and normal TSH groups, as well as low and normal-high FT4 group, according to the reference range of TSH or FT4 levels. The 90-day mortality and critical illness rates were compared among patients with low and normal TSH levels, as well as among patients with low FT4 levels and normal-high FT4 levels; in addition, differences in demographic and laboratory data were compared. A Kaplan-Meier analysis and Cox proportional hazards models were used to assess the associations of TSH and FT4 levels with mortality.

Results: One hundred fifty patients with low FT3 levels and without a history of thyroid disease were included, 68\% of whom had normal FT4 and TSH levels. Critical illness rates $(74.07 \%$ VS $37.40 \%, P=0.001)$ and mortality rates (51.85\% VS 22.76\%, $P=0.002$ ) were significantly higher in the low TSH group than in the normal TSH group. Although no significant difference in the critical illness rate was found $(P=0.296)$, the mortality rate was significantly higher in the low FT4 group $(P=0.038)$. Low TSH levels were independently related to 90-day mortality (hazard ratio $=2.78,95 \%$ Cl: $1.42-5.552, P=0.003)$.
\end{abstract}

\footnotetext{
* Correspondence: tjlaojing@163.com; felu@tjh.tjmu.edu.cn

${ }^{\dagger}$ Jing-bin Li and Fu-er Lu contributed equally to this work.

'Department of Integrated Traditional Chinese and Western Medicine, Tongji

Hospital, Tongji Medical College, Huazhong University of Science and

Technology, Wuhan, China

${ }^{2}$ Institute of Integrated Traditional Chinese and Western Medicine, Tongji

Hospital, Tongji Medical College, Huazhong University of Science and

Technology, Wuhan, China

Full list of author information is available at the end of the article
}

(c) The Author(s). 2021 Open Access This article is licensed under a Creative Commons Attribution 4.0 International License, which permits use, sharing, adaptation, distribution and reproduction in any medium or format, as long as you give appropriate credit to the original author(s) and the source, provide a link to the Creative Commons licence, and indicate if changes were made. The images or other third party material in this article are included in the article's Creative Commons licence, unless indicated otherwise in a credit line to the material. If material is not included in the article's Creative Commons licence and your intended use is not permitted by statutory regulation or exceeds the permitted use, you will need to obtain permission directly from the copyright holder. To view a copy of this licence, visit http://creativecommons.org/licenses/by/4.0/. The Creative Commons Public Domain Dedication waiver (http://creativecommons.org/publicdomain/zero/1.0/) applies to the data made available in this article, unless otherwise stated in a credit line to the data. 
Conclusions: Low FT4 and TSH concentrations were associated with mortality in patients with COVID-19 presenting with NTI; moreover, low TSH levels were an independent risk factor for mortality in these patients.

Keywords: COVID-19, Thyroid, Non-thyroidal illness syndrome, Mortality

\section{Background}

The outbreak of COVID-19 has posed a serious health threat globally. It plays an important role in disease prevention and control to elucidate the clinical features of COVID-19, identify prognostic predictors and explore potential treatment methods. A study from China found that approximately $27.52 \%$ of patients with COVID-19 had non-thyroidal illness syndrome (NTI) [1], which is characterized by a decrease in triiodothyronine (T3) levels and/or a decrease in thyroxin (T4) levels without an increase in thyroid-stimulating hormone (TSH) levels $[2,3]$. NTI typically occurs in patients admitted to intensive care units (ICUs) and is closely associated with the disease condition and prognosis $[3,4]$. The abovementioned study found that patients with NTI had up to 2.5-fold increased ratio for severe COVID-19 [1].

Low free T3 (FT3) levels in patients with NTI are typically related to a worse prognosis [5]. For example, low T3 syndrome was found to be a strong prognostic predictor of death in patients with cardiac disorders [6]. In addition to FT3 levels, free T4 (FT4) or TSH levels might affect the mortality of patients with NTI [7]. A study reported a worse prognosis for patients with sepsis and NTI presenting low T3 and T4 levels compared with those with low T3 levels alone [8]. According to another study from Turkey, low FT3 levels and increased FT4 levels are considered independent predictors of longterm mortality risks in chronic patients with NTI [9]. Clinically, patients with COVID-19 presenting with low FT3 and TSH levels appear to have a higher mortality rate, which is not completely consistent with previous NTI studies. Researchers have not clearly determined whether FT4 and TSH levels associated with NTI are related to the prognosis of patients with COVID-19. The clinical characteristics of NTI in patients with COVID19 need further elaboration. This study retrospectively analysed the clinical characteristics of NTI and explored whether TSH or FT4 levels were independent risk factors for mortality of hospitalized patients with both COVID-19 and NTI.

\section{Methods}

\section{Patients}

This retrospective cohort study was conducted according to STROBE guidelines. Data were collected from hospitalized patients who were admitted to Tongji Hospital between January and March 2020. By searching electronic medical records, data, including patient age, sex, medical history, disease onset time, discharge or death time, thyroid function, blood cell analysis and other inflammatory parameters, were included in the analysis. Data collection was performed before 20 April, and the data used were anonymized before use. The inclusion criteria were as follows: 1) patients with a confirmed COVID-19 diagnosis who underwent thyroid function examinations, 2) no previous thyroid disease, and 3) FT3 levels below the reference range without TSH levels above the reference range. The reference range for FT3 levels is $3.1-6.8 \mathrm{pmol} / \mathrm{L}$, the reference range for FT4 levels is $12-22 \mathrm{pmol} / \mathrm{L}$, and the reference range for TSH levels is $0.27-4.2 \mu \mathrm{IU} / \mathrm{mL}$. The reference range of thyroid function in the manual of Roche manufacturers was adopted according to after verification in healthy subjects.

\section{Endpoints}

The primary endpoints were 90-day death events and critical illness events. According to the disease severity guidelines formulated by the National Health Commission of China, namely, the "New Coronavirus-Infected Pneumonia" Severe and Critical Diagnosis and Treatment Program (Second trial version, Number 2020-127) [10], patients were classified as mild, severe and critical cases. Severe cases were defined as a blood oxygen saturation less than $93 \%$ or respiratory frequency greater than 30 in the resting state; critical cases were designated as patients who required mechanical ventilation.

\section{Examination method}

Examinations were conducted by the clinical laboratory of Tongji Hospital. Blood FT3, FT4 and TSH levels were tested using a Roche electrochemiluminescence method. Blood cells, including white blood cells (WBC) and leukomonocytes (LC), were detected using fluorescence staining and flow cytometry. Ultrasensitive C-reactive protein (CRP) levels were measured using immunoturbidimetry. Procalcitonin (PCT) levels were determined using a Roche electrochemiluminescence assay, and ferroprotein levels were measured using a Roche granuleenhanced immunoturbidimetry, Siemens chemiluminescence or Roche electrochemiluminescence method. Alanine aminotransferase (ALT) and aspartate aminotransferase (AST) levels were determined using the Roche kinetic method. Lactate dehydrogenase (LDH) levels were detected using a Roche colorimetric assay, and cardiac troponin I (cTnI) levels were measured 
using a Roche chemiluminescence microparticle immunoassay. Creatinine ( $\mathrm{Cr}$ ) and urea nitrogen (UREA) levels were analysed using a Roche urease assay. D dimer (D2D) levels were detected using STAGO immunoturbidimetry. Clinical and laboratory data were included at the time point of the thyroid function examination. If patients had more than one thyroid test during their hospitalization, only the first result was included in the analysis.

\section{Statistical analysis}

For descriptive variables, continuous data are presented as the medians and interquartile ranges (IQRs), and categorical data are presented as counts and percentages. A $P$ value less than 0.05 indicated a significant difference. The chi-square test was performed to compare the proportions of critical illness and death among patients with different FT4 levels or with different TSH levels; Fisher's exact test was used for a frequency $<5$. According to the reference range, patients were divided into low TSH $(<$ $0.27 \mu \mathrm{IU} / \mathrm{mL})$ and normal TSH $(0.27-4.2 \mu \mathrm{IU} / \mathrm{mL})$ groups or low FT4 $(<12 \mathrm{pmol} / \mathrm{L})$ and normal-high FT4 $(\geq 12 \mathrm{pmol} / \mathrm{L})$ groups. The Mann-Whitney test was used to compare the differences in blood cell counts, history of encephalopathy, levels of inflammatory factors and other biochemical parameters between groups. The Kaplan-Meier analysis and log-rank test were employed to assess the probability of cumulative 90-day mortality. Cox proportional hazards models were used to explore whether TSH levels were associated with the prognosis. Analyses were performed using SPSS 20.0 software.

\section{Results}

\section{Clinical features of the included patients}

Nine hundred forty-five patients with COVID-19 received thyroid function examinations. Among them, 150 patients had no history of thyroid disease and had FT3 levels $<3.1 \mathrm{pmol} / \mathrm{L}$ and TSH levels $\leq 4.2 \mu \mathrm{IU} / \mathrm{mL}$. Of the 150 included patients, 69 were female and 81 were male, and the median age was 69.5 years (IQR: 61-79 years; Range: 32-92 years). Regarding disease severity, 66 patients (44\%) were critically ill, 59 patients $(39.33 \%)$ were seriously ill, 25 patients (16.67\%) were mildly ill, and 46 patients (30.67\%) died. None of the patients had a history of pituitary gland disease.

\section{Profiles of FT4 and TSH levels among included patients with low FT3 levels}

As shown in Table 1, among patients with low serum FT3 levels, 68\% had normal FT4 and TSH levels. Additionally, 14\% had normal FT4 and decreased TSH levels. The proportion of patients with decreased FT4 levels and normal TSH levels was 9.33\%. Approximately 4.67\%
Table 1 Numbers and ratio of different FT4 and TSH levels in patients with COVID-19 and low FT3 levels

\begin{tabular}{lllll}
\hline \multicolumn{5}{c}{ FT4 } \\
\cline { 3 - 5 } & & $>22 \mathrm{pmol} / \mathrm{L}$ & $12-22 \mathrm{pmol} / \mathrm{L}$ & $<12 \mathrm{pmol} / \mathrm{L}$ \\
\hline TSH & $0.27-4.2 \mathrm{ulU} / \mathrm{mL}$ & $7(4.67 \%)$ & $102(68 \%)$ & $14(9.33 \%)$ \\
& $<0.27 \mathrm{ulU} / \mathrm{mL}$ & $4(2.67 \%)$ & $21(14 \%)$ & $2(1.33 \%)$ \\
\hline FT3 free triiodothyronin, FT4 free thyroxine & TSH thyroid stimulating hormone
\end{tabular}

of patients had elevated FT4 and normal TSH levels. Few patients had reduced TSH and abnormal FT4 levels.

\section{Comparison between the low TSH and normal TSH groups}

The patients were divided into a low TSH group and a normal TSH group according to whether the TSH level was within the reference range. Regarding baseline features, no significant differences were observed in age or sex between the low TSH and normal TSH groups. As shown in Table 2, critical illness and mortality rates were significantly higher in the low TSH group than in the normal TSH group.

To explore the possible causes or relevant factors of decreased TSH, the history of encephalopathy, biochemical markers (ALT, AST, ALB, LDH, Cr, UREA and cTnI), blood cell (WBC and LC) counts, inflammatory parameters (CRP, ferroprotein and $\mathrm{PCT}$ ), and $\mathrm{D}$ dimer (D2D) levels were compared between the low and normal TSH groups. As shown in Table 2, significant differences between the two groups were observed in inflammatory parameters, including WBC counts $(P=$ 0.023), CRP levels $(P=0.013)$ and ferroprotein levels $(P=0.047)$. In addition, significant differences in AST $(P=0.042)$, LDH $(P=0.038)$, and UREA levels $(P<$ $0.001)$ were also detected. However, no significant differences were found in the history of encephalopathy $(P=$ $0.551)$, LC $(P=0.572)$, PCT $(P=0.084)$, D2D $(P=0.316)$, ALT $(P=0.219)$, ALB $(P=0.605)$, Cr $(P=0.194)$ or cTnI $(P=0.098)$ levels. In addition, no significant difference was found in FT3 and FT4 levels between the low TSH group and the normal TSH group.

\section{Comparison between the low FT4 and normal-high FT4 groups}

The patients were also divided into a low FT4 group and a normal-high FT4 group according to whether the FT4 level was below the reference range. No significant differences were observed between the two groups in age and gender (Table 3). Although a significant difference in the critical illness rate was not identified, the mortality rate was significantly higher in the low FT4 group $(P=0.038)$.

Significant differences were observed in PCT $(P=$ $0.021)$, AST $(P=0.025)$ and $\mathrm{Cr}(P=0.029)$ levels between 
Table 2 Comparison between low TSH and normal TSH groups in patients with COVID-19 presenting with NTI

\begin{tabular}{|c|c|c|c|c|}
\hline & $\begin{array}{l}\text { Overall } \\
(n=150)\end{array}$ & $\begin{array}{l}\text { Low TSH } \\
(n=27)\end{array}$ & $\begin{array}{l}\text { Normal TSH } \\
(n=123)\end{array}$ & $P$ value \\
\hline Age, y, M (IQR) & $69.5(61,79)$ & $68(62.5,78)$ & $70(60.5,79)$ & 0.924 \\
\hline Gender (female n, \%) & $69(46 \%)$ & $12(44.44 \%)$ & $57(46.34 \%)$ & 0.858 \\
\hline Critical illness (n, \%) & $66(44 \%)$ & $20(74.07 \%)$ & $46(37.40 \%)$ & 0.001 \\
\hline Mortality (n, \%) & $42(28 \%)$ & $14(51.85 \%)$ & $28(22.76 \%)$ & 0.002 \\
\hline History of cerebropathy (n, \%) & $17(11.33 \%)$ & $4(14.81 \%)$ & $13(10.57 \%)$ & 0.551 \\
\hline WBC, ${ }^{*} 10 \wedge 9 / L, M(I Q R)$ & $10.73(7.795,16.795)$ & $14.08(10.46,21.2)$ & $10.2(7.19,16.37)$ & 0.023 \\
\hline LC, ${ }^{*} 10 \wedge 9 / \mathrm{L}, \mathrm{M}(\mathrm{IQR})$ & $1.45(0.945,1.87)$ & $1.13(0.82,1.97)$ & $1.45(0.985,1.87)$ & 0.572 \\
\hline CRP, mg/L, M (IQR) & $91.2(41.825,177.275)$ & $162.6(70.9,224.9)$ & $84(36.45,155.7)$ & 0.013 \\
\hline Ferroprotein, ug/L, M (IQR) & $1314.7(631.92395 .6)$ & $1879.35(1042.83,2861.8)$ & $1166.2(562.35,2147.15)$ & 0.047 \\
\hline$P C T, n g / m L, M(I Q R)$ & $0.19(0.08,0.99)$ & $0.39(0.115,4.16)$ & $0.17(0.08,0.86)$ & 0.084 \\
\hline ALT, U/L, M (IQR) & $44.5(22.25,73.75)$ & $57(29,69.5)$ & $42(21,76)$ & $0.219)$ \\
\hline AST, U/L, M (IQR) & $44.5(26,75.75)$ & $57(37.5,109.5)$ & $42(25,69.5)$ & 0.042 \\
\hline ALB, g/L, M (IQR) & $35.05(30.175,39.15)$ & $36.3(33.5,39.3)$ & $34.9(30.15,38.8)$ & 0.605 \\
\hline LDH, U/L, M (IQR) & $221(180,282)$ & $279(188,413)$ & $216.5(174,271.5)$ & 0.038 \\
\hline CR, umol/L, M (IQR) & $87(68.25,117.75)$ & $88(76,184)$ & $87(67,114.5)$ & 0.194 \\
\hline UREA, mmol/L, M (IQR) & $8.9(5.7,15.85)$ & $14.1(9.7,26.35)$ & $7.6(5.2,14.2)$ & $<0.001$ \\
\hline cTnl, pg/mL, M (IQR) & $18.05(6.35,197.875)$ & $70.7(11.2,755.65)$ & $13.8(6.2,162.7)$ & 0.098 \\
\hline D2D, ug/mL FEU, M (IQR) & $5.365(1.46,19.045)$ & $8.41(2.47,17.74)$ & $4.5(1.39,19.06)$ & 0.316 \\
\hline FT3, pmol/L, M (IQR) & $2.665(2.303,2.888)$ & $2.68(2.35,2.92)$ & $2.66(2.31,2.88)$ & 0.696 \\
\hline FT4, pmol/L, M (IQR) & $16.05(13.623,18.345)$ & $16.39(13.53,18.89)$ & $16(13.75,18.25)$ & 0.687 \\
\hline TSH, ulU/mL, M (IQR) & $0.96(0.412,1.848)$ & $0.16(0.10,0.21)$ & $1.29(0.69,2.03)$ & / \\
\hline
\end{tabular}

NTI non-thyroidal illness syndrome, $M$ (IQR) Median (interquartile range), WBC white blood cell, $L C$ leukomonocyte, $C R P$ ultrasensitive $C$-reactive protein, $P C T$ procalcitonin, $A L T$ alanine aminotransferase, AST aspertate aminotransferase, $A L B$ albumin, $L D H$ lactate dehydrogenase, $C r$ Creatinine, UREA urea nitrogen, $C T n I$ cardiac troponin I, D2D D dimmers

the low FT4 group and the normal-high FT4 group. However, no significant differences were found in WBC counts $(P=0.455)$ LC $(P=0.284), \mathrm{CRP}(P=0.866)$ and ferroprotein $(P=0.214)$, ALT $(P=0.976)$, ALB $(P=$ $0.277)$, LDH $(P=0.066)$, UREA $(P=0.06)$, cTnI $(P=$ $0.189)$, and $\mathrm{D} 2 \mathrm{D}(P=0.479)$ levels. In addition, relatively lower FT3 levels were detected in the low FT4 group, while no significant difference in TSH levels was observed between the low FT4 and normal-high FT4 groups.

\section{Association between TSH or FT4 levels and 90-day mortality}

The probability of 90-day mortality was assessed using a Kaplan-Meier analysis (Fig. 1). The mortality rate was significantly different between the low TSH group and the normal TSH group (log-rank $P=0.001)$, but it was not significantly different between the low FT4 group and the normal-high FT4 group (log-rank $P=0.174$ ).

The Cox proportional hazards model (Table 4) suggested that low TSH levels were independently related to 90 -day mortality (hazard ratio $=2.78$, 95\% CI: $1.42-$ 5.552, $P=0.003)$. Low TSH levels were an independent risk factor for mortality in patients with both COVID-19 and NTI.

\section{Discussion}

NTI refers to alterations in a thyroid test without thyroid lesions in individuals with acute or chronic nonthyroid diseases [6], and it has been reported in many diseases, such as fractures, major trauma, cardiac surgery, meningococcal septic shock, community-acquired pneumonia, kidney disease, lymphocytic leukemia, and diffuse large B cell lymphoma [3]. NTI is characterized by decreased T3 levels and no elevation in TSH levels, always with increased rT3 levels [3]. High, normal or low T4 levels, as well as low or normal TSH levels, have been observed in patients with NTI [3], but the corresponding proportions were unclear. In this study, most the included patients with COVID-19 presenting low FT3 levels had normal FT4 and TSH levels, and then $14 \%$ had normal FT4 levels and decreased TSH levels. Although the lung is the key lesioned organ, angiotensin-converting enzyme 2 (ACE2), the receptor for SARS-CoV-2 to invade humans, is expressed at a high level in the thyroid $[11,12]$. Many cases of subacute 
Table 3 Comparison between low and normal-high FT4 groups in patients with COVID-19 presenting with NTI

\begin{tabular}{|c|c|c|c|}
\hline & $\begin{array}{l}\text { Low FT4 } \\
(n=16)\end{array}$ & $\begin{array}{l}\text { Normal-high FT4 } \\
(n=134)\end{array}$ & $P$ value \\
\hline Age, y, M (IQR) & $64.5(41.75,71.75)$ & $70(62.25,79.75)$ & 0.077 \\
\hline Gender (female n, \%) & $8(50 \%)$ & $61(45.52 \%)$ & 0.734 \\
\hline Critical illness (n, \%) & 9 (56.25\%) & $57(42.54 \%)$ & 0.296 \\
\hline Mortality (n, \%) & $8(50 \%)$ & $34(25.37 \%)$ & 0.038 \\
\hline WBC, ${ }^{*} 10 \wedge 9 / L, M(I Q R)$ & $10.35(8.34,26.87)$ & $10.92(7.79,16.65)$ & 0.455 \\
\hline LC, *10^9/L, M (IQR) & $1.175(0.72,1.75)$ & $1.48(0.98,1.87)$ & 0.284 \\
\hline CRP, mg/L, M (IQR) & $71.75(37.65,225.1)$ & $95.3(42.675,172.2)$ & 0.886 \\
\hline Ferroprotein, ug/L, M (IQR) & $1782.2(767.95,7462.3)$ & $1221.9(618.6,2303.05)$ & 0.214 \\
\hline$P C T, n g / m L, M(I Q R)$ & $0.97(0.15,8.56)$ & $0.17(0.08,0.87)$ & 0.021 \\
\hline ALT, U/L, M (IQR) & $47(23.75,76)$ & $43(21.25,73.75)$ & 0.976 \\
\hline AST, U/L, M (IQR) & $91.5(38.25,221.25)$ & $43(26,68.75)$ & 0.025 \\
\hline ALB, g/L, M (IQR) & $32(29.55,34.55)$ & $35.7(30.7,39.3)$ & 0.277 \\
\hline LDH, U/L, M (IQR) & $347(248,405)$ & $216.5(177,272.5)$ & 0.066 \\
\hline CR, umol/L, M (IQR) & $124.5(82.5,214.5)$ & $87(66.5,114)$ & 0.029 \\
\hline UREA, mmol/L, M (IQR) & $17.9(5.73,28.5)$ & $8.55(5.7,14.33)$ & 0.06 \\
\hline cTnl, pg/mL, M (IQR) & $287(8.7,803.4)$ & $15.9(6.4,139)$ & 0.189 \\
\hline D2D, ug/mL FEU, M (IQR) & $7.51(1.85,21)$ & $5.24(1.39,18.94)$ & 0.479 \\
\hline FT3, pmol/L, M (IQR) & $2.36(1.8,2.795)$ & $2.69(2.3625,2.91)$ & 0.027 \\
\hline FT4, pmol/L, M (IQR) & $10.55(9.01,11.69)$ & $16.75(14.38,18.46)$ & / \\
\hline TSH, ulU/mL, M (IQR) & $1.82(0.62,2.15)$ & $0.897(0.41,1.79)$ & 0.235 \\
\hline
\end{tabular}

thyroiditis after SARS-CoV-2 infection have been reported $[13,14]$. Presumably, NTI was combined with thyrotoxicosis in some patients with COVID-19, especially in those with low FT3 and elevated FT4 levels.

The prognostic effects of NTI on critically ill patients have been extensively studied and recognized, but associations of altered thyroid tests and mortality in patients with NTI are contradictory. In a study focusing on patients with sepsis presenting with NTI, a worse prognosis was observed for patients with combined low T3 and T4 levels compared with those with low T3 levels alone [8]. In a study from Turkey, low FT3 levels and increased FT4 levels were found to be independent predictors of long-term mortality risks in chronic patients with

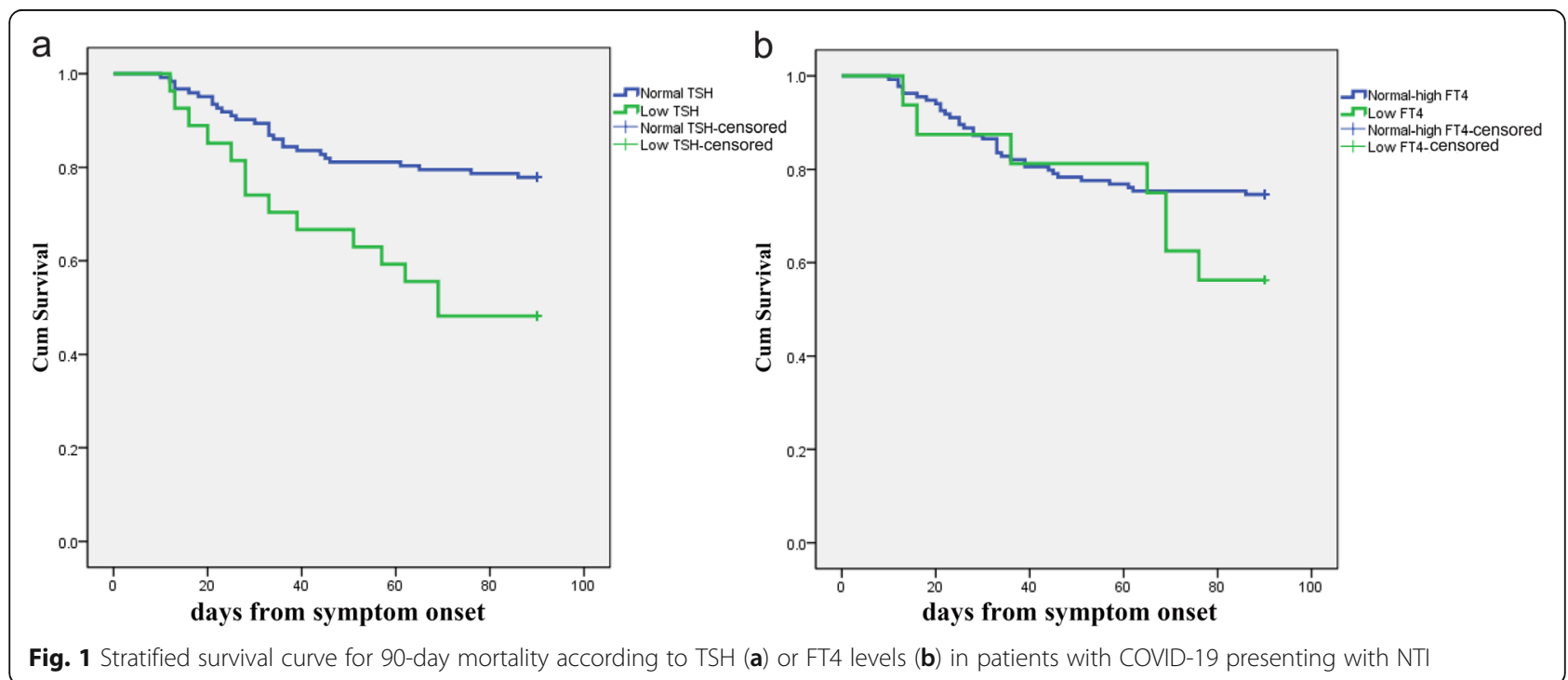


Table 4 Association of low TSH and mortality in the cox regression analysis model

\begin{tabular}{llllllll}
\hline & Beta & SE & Wald & $\begin{array}{l}\boldsymbol{P} \\
\text { value }\end{array}$ & $\begin{array}{l}\text { Hazard } \\
\text { ratio }\end{array}$ & \multicolumn{2}{l}{$\mathbf{9 5 . 0 \%} \mathrm{Cl}$} \\
\cline { 7 - 9 } & & & & & Lower & Upper \\
\hline TSH & 1.010 & 0.342 & 8.738 & 0.003 & 2.745 & 1.405 & 5.363 \\
gender & 0.174 & 0.325 & 0.288 & 0.591 & 1.190 & 0.630 & 2.250 \\
age & 0.619 & 0.347 & 3.185 & 0.074 & 1.858 & 0.941 & 3.668 \\
CRP & 1.308 & 0.736 & 3.155 & 0.076 & 3.699 & 0.873 & 15.665 \\
FT4 & 0.710 & 0.420 & 2.859 & 0.091 & 2.034 & 0.893 & 4.635 \\
\hline
\end{tabular}

NTI, and TSH levels were not significantly associated with mortality [9]. Another study in Germany found that high TSH, T3 and T4 levels were related to a better prognosis in patients with acute liver failure [15].

For patients with COVID-19, NTI has been found to be significantly associated with the disease condition [1]. In a study conducted in Italy, Ilaria Muller's team compared patients with and without COVID-19 in a high ICU, and no significant difference was observed in FT3 levels, indicating that the NTI rate might not be increased for patients with COVID-19 treated in the ICU [16]. Lower TSH levels and higher FT4 concentrations were also detected in patients with COVID-19 admitted to the high ICU compared with patients with COVID-19 admitted to the low ICU [16]. The study by Min Chen et al analysed thyroid function in patients with COVID19. Lower TSH and FT3 concentrations were shown to be associated with the severity of COVID-19 $(p<0.001)$, and TSH levels were significantly lower in severely and critically ill patients with COVID-19 than in nonCOVID-19 control patients [7]. In the study conducted by Lania et al., the mortality was higher and the duration of hospitalization was longer in thyrotoxic in-hospital patients with COVID-19 as compared to those with normal thyroid function [17]. Here, mortality and critical illness rates were significantly higher in the low TSH group than in the normal TSH group, and low TSH levels were independently related to 90-day mortality (hazard ratio $=2.78, P=0.003$ ). In addition, the mortality rate was significantly higher in the low FT4 group, but no significant difference in the critical illness rate was found. Although thyrotoxicosis might occur in patients with COVID-19, low FT3-FT4 levels or low FT3-TSH levels suggested a worse prognosis. Critically, severely and mildly ill patients with COVID-19 complicated with NTI were all included in this study, and the analysis of the effects of TSH and FT4 levels on the prognosis was based on low FT3 levels, which partially explained the difference in our results from other findings.

What is the mechanism of low-TSH in patients with COVID-19 and NTI? Why low FT3-TSH level might affect the mortality in patients with COVID-19? Significant differences were observed in the levels of nonspecific inflammatory parameters between the low TSH and normal TSH groups, including WBC counts and CRP and ferroprotein levels. Presumably, inflammation suppresses the hypothalamic-pituitary-thyroid axis [18], which might be one cause of low TSH levels in patients with COVID-19 presenting with NTI [19]. Researchers have not clearly determined whether SARS$\mathrm{CoV}-2$ invades the pituitary gland and damages TSHsecreting cells [7]. In addition, significant differences in AST, LDH, and UREA levels were observed between the low TSH and normal TSH groups of patients with both COVID-19 and NTI, and prospective clinical and basic studies are needed to explore whether these alterations are the cause of low TSH levels associated with NTI in patients with COVID-19. The deteriorative feedback regulation of pituitary-thyroid axis and the concomitant injuries of other organs may partially explain the mortality, but the pathophysiological mechanism needs further study.

This retrospective study has several limitations. Examinations of rT3 levels, ultrasound inspections of the thyroid and specific antibody tests were not available. In addition, information about steroid and dopamine therapy was unaccessible before hospitalization, and medication data were not collected and analysed. Further studies are needed to determine whether thyroxine supplementation is beneficial based on alterations in thyroid tests.

\section{Conclusions}

Low FT4 and TSH concentrations were associated with mortality in patients with both COVID-19 and NTI; moreover, low TSH levels were an independent risk factor for mortality in these patients.

\section{Abbreviations \\ NTI: non-thyroidal illness syndrome; FT3: free triiodothyronine; FT4: free thyroxine; TSH: thyroid stimulating hormone; ICU: intensive care units; WBC: white blood cell; LC: leukomonocyte; CRP: ultrasensitive C-reactive pro- tein; PCT: procalcitonin; ALT: alanine aminotransferase; AST: aspertate aminotransferase; ALB: albumin; LDH: lactate dehydrogenase; cTnl: cardiac troponin I; Cr: Creatinine; UREA: urea nitrogen; D2D: D dimmers; \\ ACE2: Angiotensin-converting enzyme 2}

\section{Acknowledgments}

Thanks to all people who fight against COVID-19.

\section{Authors' contributions}

$J G, F L, D W$, and $J L$ designed the study and had full access to the data in the study and take responsibility for the integrity of the data and the accuracy of the data analysis. HD, QX, ZH, JL, YZ, XC, and FY helped to collect the data, do the analysis, and interpret the data. All authors approved the version to be published.

\section{Funding}

This study is supported by the National Natural Science Foundation of China (81904158, 81974567 and 81874382). The funders played no roles in the design, data collection and analysis of the study and the preparation of this manuscript. 


\section{Availability of data and materials}

Data were available through contacting the corresponding authors.

\section{Declarations}

Ethics approval and consent to participate

The study was approved by the ethical committee of Tongji Hospital, Tongji Medical College, Huazhong University of Science and Technology. Informed consent was waived by the ethical commission.

\section{Consent for publication}

Not applicable.

\section{Competing interests}

We declare no competing interests.

\section{Author details}

'Department of Integrated Traditional Chinese and Western Medicine, Tongji Hospital, Tongji Medical College, Huazhong University of Science and Technology, Wuhan, China. ${ }^{2}$ Institute of Integrated Traditional Chinese and Western Medicine, Tongji Hospital, Tongji Medical College, Huazhong University of Science and Technology, Wuhan, China. ${ }^{3}$ Department of Clinical Laboratory, Tongji Hospital, Tongji Medical College, Huazhong University of Science and Technology, Wuhan, China. ${ }^{4}$ China Clinical Research Center, Tongji Hospital, Tongji Medical College, Huazhong University of Science and Technology, Wuhan, China.

Received: 23 December 2020 Accepted: 13 May 2021

Published online: 27 May 2021

\section{References}

1. Zou R, Wu C, Zhang S, Wang G, Zhang Q, Yu B, et al. Euthyroid sick syndrome in patients with COVID-19. Front Endocrinol. 2020;11:566439.

2. Langouche $L$, Jacobs A, Van den Berghe G. Nonthyroidal illness syndrome across the ages. J Endocr Soc. 2019;3(12):2313-25.

3. Fliers $E$, Bianco AC, Langouche $L$, Boelen A. Thyroid function in critically ill patients. Lancet Diabetes Endo. 2015:3(10):816-25.

4. Lado-Abeal J, Diaz C, Berdine G, Iwuji K, Araujo-Vilar D, Lampon-Fernandez $\mathrm{N}$, et al. High prevalence of non-thyroidal illness syndrome in patients at long-term care facilities. Endocrine. 2020;70(2):348-55.

5. Gao W, Guo W, Guo Y, Shi M, Dong G, Wang G, et al. Thyroid hormone concentrations in severely or critically ill patients with COVID-19. J Endocrinol Invest. 2021;44(5):1031-40.

6. Van den Berghe G. Non-thyroidal illness in the ICU: a syndrome with different faces. Thyroid. 2014;24(10):1456-65.

7. Chen M, Zhou W, Xu W. Thyroid function analysis in 50 patients with COVID-19: A retrospective study. Thyroid. 2021;31(1):8-11.

8. Padhi R, Kabi S, Panda BN, Jagati S. Prognostic significance of nonthyroidal illness syndrome in critically ill adult patients with sepsis. Int J Crit Illn Inj Sci. 2018;8(3):165-72.

9. Ataoğlu HE, Ahbab S, Serez MK, Yamak M, Kayaş D, Canbaz ET, et al. Prognostic significance of high free T4 and low free T3 levels in nonthyroidal illness syndrome. Eur J Intern Med. 2018;57:91-5.

10. National Health Commission of China: the "New Coronavirus-Infected Pneumonia" Severe and Critical Diagnosis and Treatment Program, (Second trial version, Number 2020-127).2020.4. http://www.nhc.gov.cn/yzygj/s7653 p/202004/c083f2b0e7eb4036a59be419374ea89a.shtml.. Accessed 04 Oct 2020.

11. Li MY, Li L, Zhang Y, Wang XS. Expression of the SARS-CoV-2 cell receptor gene ACE2 in a wide variety of human tissues. Infect Dis Poverty. 2020;9(1): 45.

12. Scappaticcio L, Pitoia F, Esposito K, Piccardo A, Trimboli P. Impact of COVID19 on the thyroid gland: an update. Rev Endocr Metab Disord. 2020:1-13.

13. Brancatella A, Ricci D, Viola N, Sgrò D, Santini F, Latrofa F. Subacute Thyroiditis After Sars-COV-2 Infection. J Clin Endocrinol Metab. 2020;105(7): dgaa276.

14. Ippolito S, Dentali F, Tanda ML. SARS-CoV-2: a potential trigger for subacute thyroiditis? Insights from a case report. J Endocrinol Investig. 2020;43(8): $1171-2$.

15. Anastasiou O, Sydor S, Sowa JP, Manka P, Katsounas A, Syn WK, et al. Higher thyroid-stimulating hormone, Triiodothyronine and Thyroxine values are associated with better outcome in acute liver failure. PLoS One. 2015;10(7): e0132189.

16. Muller I, Cannavaro D, Dazzi D, Covelli D, Mantovani G, Muscatello A, et al. SARS-CoV-2-related atypical thyroiditis. Lancet Diabetes Endo. 2020;8(9):73941.

17. Lania A, Sandri MT, Cellini M, Mirani M, Lavezzi E, Mazziotti G. Thyrotoxicosis in patients with COVID-19: the THYRCOV study. Eur J Endocrinol. 2020; 183(4):381-7.

18. McElvaney OJ, McEvoy NL, McElvaney OF, Carroll TP, Murphy MP, Dunlea DM, O NC, Clarke J, O'Connor E, Hogan G et al: Characterization of the inflammatory response to severe COVID-19 illness. Am J Resp Crit Care 2020; 202(6):812-821.

19. Pal R. COVID-19, hypothalamo-pituitary-adrenal axis and clinical implications. Endocrine. 2020;68(2):251-2.

\section{Publisher's Note}

Springer Nature remains neutral with regard to jurisdictional claims in published maps and institutional affiliations.
Ready to submit your research? Choose BMC and benefit from:

- fast, convenient online submission

- thorough peer review by experienced researchers in your field

- rapid publication on acceptance

- support for research data, including large and complex data types

- gold Open Access which fosters wider collaboration and increased citations

- maximum visibility for your research: over $100 \mathrm{M}$ website views per year

At BMC, research is always in progress.

Learn more biomedcentral.com/submissions 